\title{
Fiber Reinforced Adhesive Patch (FRAP): A New Technology for Minimal Invasive Treatments of Bone Fractures
}

\author{
Kyrre Pedersen ${ }^{1}$, Axel Nordberg ${ }^{2}$, Peter Halldin ${ }^{2}$ and Hans von Holst ${ }^{1,2^{*}}$ \\ ${ }^{1}$ Department of Neurosurgery, Karolinska University Hospital, Stockholm, Sweden \\ ${ }^{2}$ Division of Neuroengineering, Royal Institute of Technology, Stockholm, Sweden
}

*Corresponding author: Hans von Holst, Department of Neurosurgery, Karolinska University Hospital, 17176 Stockholm, Sweden, Tel: +46 851770000 ; E-mail: hans.vonholst@karolinska.se

Rec date: Mar 25, 2014; Acc date: Apr 21, 2014; Pub date: Apr 23, 2014

Copyright: ( 2014 Pedersen K, et al. This is an open-access article distributed under the terms of the Creative Commons Attribution License, which permits unrestricted use, distribution, and reproduction in any medium, provided the original author and source are credited.

\begin{abstract}
Instead of screws and metal plates we have developed a unique adhesive implant to stabilize the various types of bone fractures defined as the Fiber Reinforced Adhesive Patch (FRAP) technology. The new implant consists of an adhesive strengthened with fibers to form a composite patch. The FRAP technology is developed as a degradable adhesive facilitating a mechanically strong triazine system based on non-toxic allylic and thiol compounds. The thiolene cross linking strategy is highly desirable in a surgical environment as it can be performed via minimally invasive optical fibers and with excellent tolerance to oxygen. The number of layers and the size of the FRAP technology is chosen by the surgeon depending on the fracture characteristics and the anticipated load on the fracture. When at place, the tailor-made FRAP technology is photo-cured by UV light to a hard composite thereby bridging and stabilizing the fracture or the skull bone after neurosurgical operations. The experimental and numerical analysis on bovine bone fractures shows that the new FRAP technology should become an excellent alternative or complement to existing metal implants.
\end{abstract}

Keywords: Adhesive; Bone fracture; Finite element modelling

\section{Introduction}

The global annual incidence of both acute and chronic types of various bone injuries is enormous and will increase with a growing elderly population within the next decades [1,2]. Existing surgical treatment of complicated fractures in the neurosurgical and orthopedic fields mostly rely on the application of screw-fixated metal implants. Due to their rigid design, the intervention requires open surgery and general anesthesia.

Adhesive fixation of bone fractures has been an area of interest since the middle of the $20^{\text {th }}$ century as an alternative to conventional fixation with metal screws and plates [3-11]. Since adhesives do not demand drilling and can be distributed with minimal invasive surgery through an endoscope under local anaesthesia, it possesses some obvious advantages. So far no biocompatible adhesive has yet been proven to possess sufficient strength for bone fracture stabilization. A unique adhesive implant has been developed to stabilize the various types of bone fractures defined as the Fiber Reinforced Adhesive Patch (FRAP) technology. The new implant consists of an adhesive, strengthened with fibers to form a composite FRAP technology and developed as a degradable adhesive facilitating a mechanically strong two component triazine adhesive system based on non-toxic thiol and allylic compounds. The thiol-ene cross-linking strategy is highly desirable in a surgical environment as it can be performed via minimally invasive endoscopy. The intention is that the number of layers and size of the FRAP technology, implanted under local anaesthesiology, will be chosen by the surgeon depending on the fracture characteristics and the anticipated load on the fracture. However, it is unknown whether the strength of FRAP fixation is mechanically sufficient to maintain stability during daily loading after surgery. In order to set requirements for the strength of the FRAP technology, numerical analysis is a strong complement to further evaluate experimental results before use in health care. The Finite Element (FE) method is the numerical method of choice for this application as data from experiments can be further analyzed and confirmed in complex geometry and materials.

The aim of the present experimental study and numerical analysis is

- to mechanically analyse the strength of the FRAP technology on induced bovine bone fractures and

- to analyse the mechanical results on the first and second cervical vertebrae by using an FE model developed for the human cervical spine.

\section{Materials and Methods}

\section{Specimen preparation}

Fresh bovine femur bones were collected and frozen to -30 degrees Celsius. A thin layer of soft tissue was left to prevent the bone from drying during freezing. On the day of testing, the bone was thawed at room temperature and cleaned of all remaining soft tissue, including bone marrow. The hollow pipe-shaped bones were then split into eight rod-like shapes and sawed into lengths of approximately $75 \mathrm{~mm}$. Each of the rods were then wet sanded with a "120" sandpaper to achieve smooth and evenly shaped rods. To create a generic fracture, the rods were sawed into two pieces. The two pieces were then bonded with 2-6 lamina of a fiber reinforced adhesive to form a bonding patch, FRAP (Table 1). Specimens \#1-18 were designed to induce a cohesive failure mode while specimens \#19-24 were designed to fail adhesively. 
Citation: Pedersen K, Axel Nordberg A, Peter Halldin P, Holst HV, et al. (2014) Fiber Reinforced Adhesive Patch (FRAP): A New Technology for Minimal Invasive Treatments of Bone Fractures. J Trauma Treat S2: 007. doi:10.4172/2167-1222.S2-007

Page 2 of 6

\begin{tabular}{|c|c|c|c|c|}
\hline Specimen & No. of lamina & Failure type & Bonding width & Patch thickness \\
\hline $1-6$ & 2 & Cohesive & $12-13 \mathrm{~mm}$ & $0.11 \mathrm{~mm}$ \\
\hline $7-12$ & 3 & Cohesive & $12-13 \mathrm{~mm}$ & $0.16 \mathrm{~mm}$ \\
\hline $13-18$ & 4 & Cohesive & $12-13 \mathrm{~mm}$ & $0.22 \mathrm{~mm}$ \\
\hline $19-24$ & 6 & Adhesive & $12-13 \mathrm{~mm}$ & $0.35 \mathrm{~mm}$ \\
\hline
\end{tabular}

Table 1: Specimens used in this study

\section{FRAP bonding}

The FRAP bond used for fixation was made of a fiber reinforced adhesive. The adhesive used was Scotch bond XT (produced by $3 \mathrm{M}$ Espe), a commercially available dental adhesive, based on a light activated polymeric system. It consists mainly of BisGMA, HEMA, dimethacrylates, ethanol, water, photo initiator and a methacrylate, functional copolymer of polyacrylic and polyitaconic acids. The fibres used were woven 90/0 E-glass fibre mat from Porcher industries, style $106,25 \mathrm{~g} / \mathrm{m}^{2}$. The FRAP bond was applied on all fractured specimens. The FRAP consists of an inner layer of adhesive, followed by 2-6 layers of fibres, and lastly, one top coat of adhesive, all applied circumferentially around the fracture but not on the ends (Figure 1). Fibre layers were all oriented with major fibre direction along with tensile direction of the specimen. Prior to applying the inner layer of adhesive, the bonding area was rinsed, etched using phosphoric acid from 3M Espe, and rinsed again. The inner layer adhesive was applied in a few consecutive layers with a gently rubbing motion. The fiber mat was then applied as strips, approximately $12-13 \mathrm{~mm}$ wide and lengthwise along the fracture ranging between 37 and $42 \mathrm{~mm}$. The top coat of adhesive was applied until the fibers were completely saturated with adhesive. Curing was performed with curing light, Elipar 2500 from $3 \mathrm{M}$, in 400-500 $\mathrm{nm}$ range, designed for dental adhesives. Curing speed was approximately $3-4 \quad \mathrm{~cm}^{2} / \mathrm{min}$. To allow complete polymerization, the specimens were kept at room temperature for 4 hours after bonding. The cortical bone was kept moist by regularly spraying it with saline.
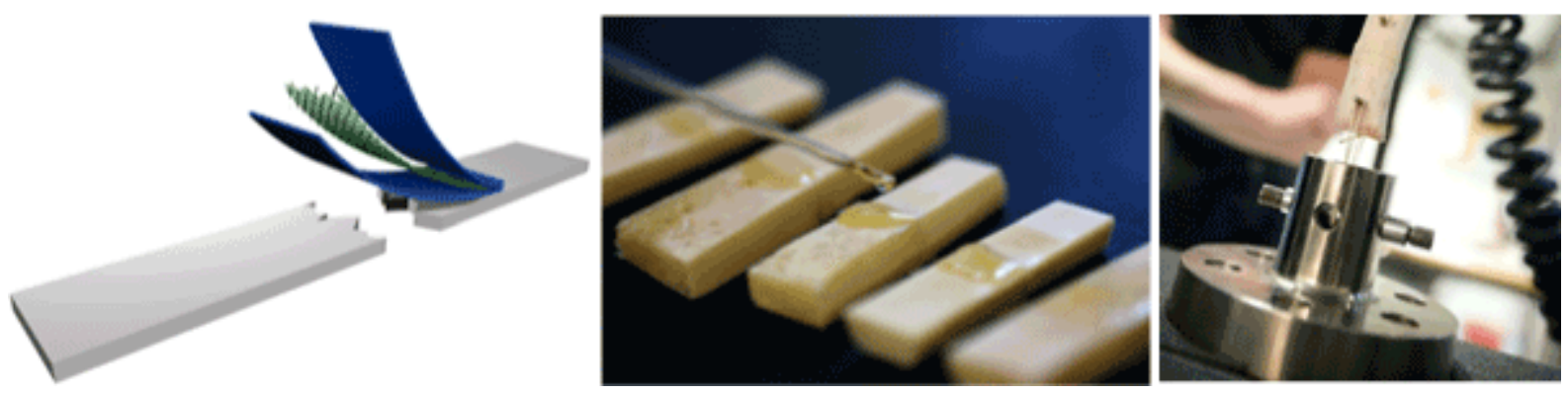

Figure 1: Schematic presentation of the FRAP technology (left) applied on the induced bovine bone fracture (middle) before mechanically tested in the Instron machine (right)

\section{Specimen testing}

All mechanical tests were performed in an Instron 5567 with a $5 \mathrm{kN}$ load cell, and with a crosshead speed of $2 \mathrm{~mm} / \mathrm{min}$. The tensile tests were performed by inserting two parallel pins through the bone with wire connections to the load cell. A Digital Speckle Photography (DSP) with Aramis software (produced by GOM GmbH) was used to measure strain vectors in the FRAP bond during tensile testing. All specimens were tested until complete failure was observed. The circumferential bonding length of the specimen was measured with a scanner using a resolution of 118 pixels per $\mathrm{cm}$.

\section{Finite element analysis}

Simulation of fracture stability was performed by modelling both fractures and fixating bonds in the KTH neck model [12,13] (Figure 2). All simulations were performed in the FE code LS-DYNA [14]. The $\mathrm{KTH}$ FE neck model includes a rigid head [15]. The seven vertebrae (C1-C7) are modelled with linear viscoelastic material models for the cortical and trabecular bone. The two uppermost thoracic vertebrae (T1-T2) are represented by rigid cubes and joints. The intervertebral discs are modelled with membrane elements with orthotropic properties for the annulus fibres, viscoelastic solid elements for the ground substance, and solid elements with incompressible material properties for the nucleus pulpous. The facet joints are modelled as sliding contacts with friction representative of cartilage. All spinal ligaments are modelled as either non-linear tension-only springs or elastic membranes with contact definitions toward relevant tissues. The cervical musculature was included as spring elements representing the passive force of the musculature. The KTH neck model has been extensively compared to experimental data from volunteer, cadaver and specimen experiments. Relevant to this study is the good kinematic agreement of the cadaver specimen testing of the upper cervical spine in quasi-static flexion and extension. Furthermore, the KTH neck model compared well to dynamic compression-flexion experiments with human cervical spine and skull specimens performed by volunteer experiments by [12]. 


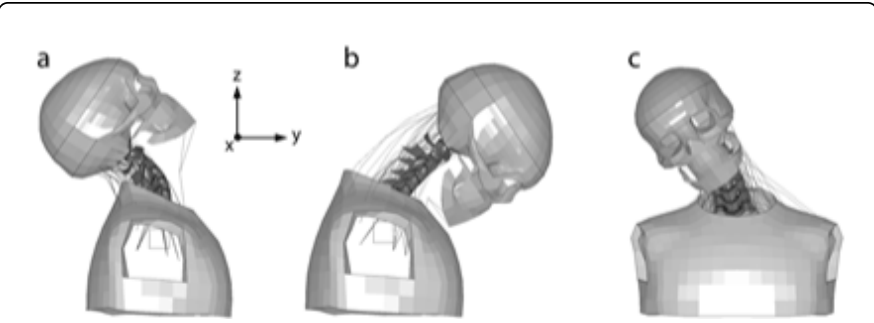

Figure 2: Motions; a) Extension with used global coordinate system, b) flexion, c) lateral bending
One $\mathrm{C} 1$ arch fracture and two $\mathrm{C} 2$ dens fractures were induced in the model, (Figure 3). The fractures were induced as a $0.4 \mathrm{~mm}$ gap in the solid cortical bone, (Figure 3c). The $\mathrm{C} 1$ fracture was a double arch fracture, also known as a Jefferson fracture. The C2 fractures were a dens type 2 fracture and a dens type 3 fracture. The FRAP was modelled with 4-node shell elements around the induced fracture, 0.2 $\mathrm{mm}$ thick and $0.4 \mathrm{~mm}$ wide. An elastic isotropic material model was used with the young modulus of $15 \mathrm{GPa}$, which was measured in the tensile experiment of the FRAP presented below). Circumferential length for the bonds were $17.1+30.0 \mathrm{~mm}$ for the double $\mathrm{C} 1$ fracture, $33.5 \mathrm{~mm}$ for the dens type $2 \mathrm{C} 2$ fracture, and $33.4 \mathrm{~mm}$ for the dens type $3 \mathrm{C} 2$ fracture. The muscles were given only passive properties.
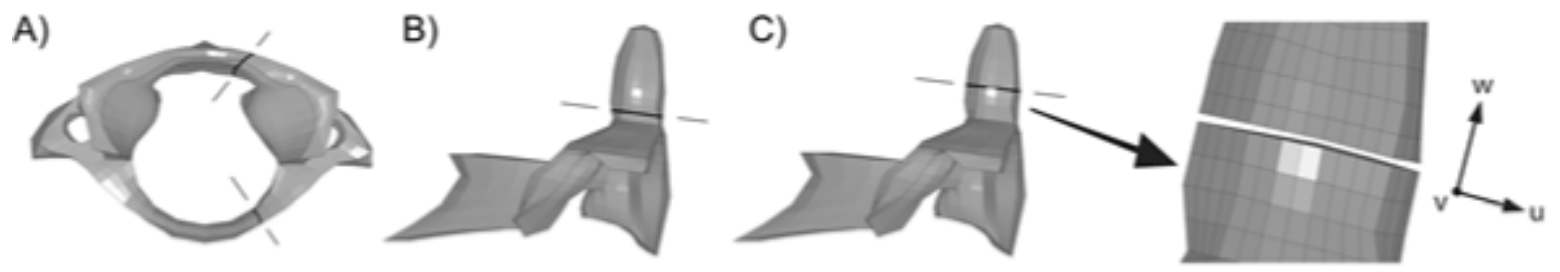

Figure 3: Bonded fractures in FE; a) Jefferson fracture, b) Dens fracture Type 3 c) Dens fracture Type 2 with local coordinate system

Six different loads were applied in order to simulate a normal flexion, extension and lateral bending in two different loading speeds. The thorax cube was rigidly fixed and a pure moment of $5 \mathrm{Nm}$ was applied to the skull. The global coordinate system used is defined in Figure 2. A local coordinate system is defined in Figure 3, where w is oriented in the tensile direction of the fixation bond. The moment was applied dynamically, as a half sinusoidal impulse, with durations of $500 \mathrm{~ms}$ and $100 \mathrm{~ms}$. Forces were computed in w direction from the isotropic shells filling the gap in the $\mathrm{C} 1$ and $\mathrm{C} 2$ simulation a FRAP.

A convergence study was done by increasing the number of elements on C2 from 2368 shells and 4992 solids to 7056 shells and 39936 solids. This also meant that the number of elements on the modelled FRAP increased from 32 to 128 . A $100 \mathrm{~N}$ load was then applied in local $u$ direction at the distal end of the dens and forces were computed in local $\mathrm{w}$ direction in the FRAP.

\section{Results}

\section{Specimen testing}

Tensile testing of bone specimen \#1-24 showed maximum FRAP bond strength, $\sigma \mathrm{m}=155 \mathrm{MPa}$ and maximum shear stress in the bonepatch interface, $\tau \mathrm{m}=6 \mathrm{MPa}$. Failure mode for specimen \#1-18 was cohesive failure and for \#19-24 adhesive failure. Young's modulus and strain to failure of the FRAP was calculated in the load direction, to $\mathrm{E}=13-15 \mathrm{GPa}$ and $\varepsilon=2.5 \%$. Figure 4 shows, for cohesively failed specimen \#1-18, maximum load divided with circumferential bond length. The size independent strength ratio $(\mathrm{N} / \mathrm{mm})$ was calculated to $34 \mathrm{~N} / \mathrm{mm}$ for the 4 lamina specimen, \#13-18, $24 \mathrm{~N} / \mathrm{mm}$ for the 3 lamina specimen, \#7-12 and $17.7 \mathrm{~N} / \mathrm{mm}$ for the 2 lamina specimen \#1-6. Maximum load for the tested specimen lay between 600 and $1260 \mathrm{~N}$, and the circumferential bonding length varied between 37 and $42 \mathrm{~mm}$. FRAP thickness was measured to $0.11 \mathrm{~mm}$ for 2 lamina specimens, $0.16 \mathrm{~mm}$ for 3 lamina specimens and $0.22 \mathrm{~mm}$ for 4 lamina specimens.

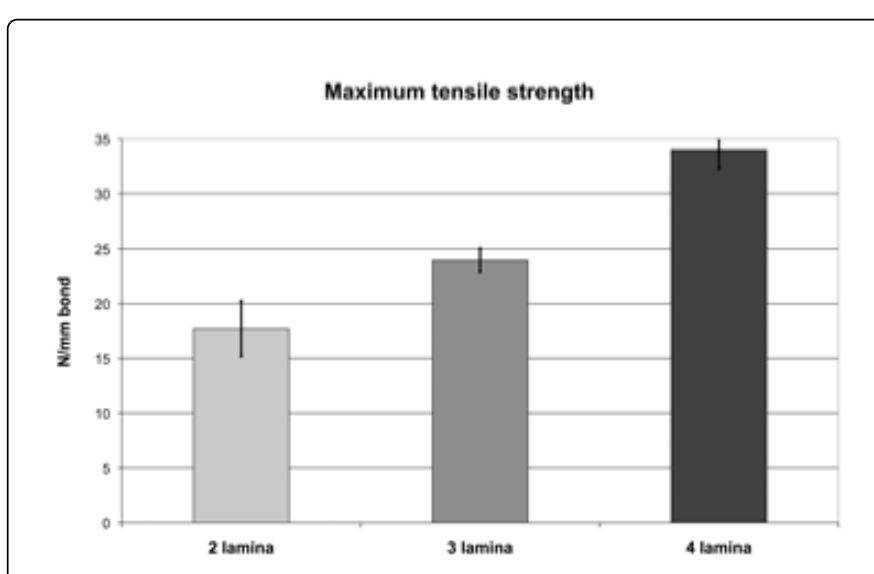

Figure 4: Measured maximum tensile strength divided by the circumferential length of the bond

\section{Finite element analysis}

From FE simulations only tensile forces in local w coordinate were considered. In order to compare the numerical results with experimental results, a dimensionless value was calculated. Measured stresses were multiplied with bonding thickness, $0.2 \mathrm{~mm}$, around the fracture, $\mathrm{N} / \mathrm{mm}$. The highest force found in the bond, $21.1 \mathrm{~N} / \mathrm{mm}$, was in the anterior part of the $\mathrm{C} 2$ dens 3 fracture during extension, (Figure 5). Maximum measured force from experiment, $34 \mathrm{~N} / \mathrm{mm}$ divided by the computed maximum force from the FE model $(21 \mathrm{~N} / \mathrm{mm})$, yields a factor of 1.61. The negative tensile force indicates a compressive load, which is transferred mainly to the adjacent bone tissue. The tensile fracture forces were generally found to be greater for the C2 dens 3 fracture than for the $\mathrm{C} 2$ dens 2 fracture, and for extension neck motion compared to flexion. It was also seen that a shorter load pulse 
Citation: Pedersen K, Axel Nordberg A, Peter Halldin P, Holst HV, et al. (2014) Fiber Reinforced Adhesive Patch (FRAP): A New Technology for

Page 4 of 6

increased fracture forces. Lateral bending showed higher forces in the C1 Jefferson fracture than the $\mathrm{C} 2$ dens fractures. All results from the FE simulations are presented in Table 2. The convergence study showed that the internal energy in the FRAP technology increased by $2.51 \%$, when increasing the number of elements in the FRAP technology from 32 to 128 .

\section{Discussion}

This study has shown that it is possible to stabilize cervical bone fractures on $\mathrm{C} 1$ and $\mathrm{C} 2$ with the FRAP technology. The bonded fractures can withstand forces higher than computed forces in an FE model of the human neck. When comparing FRAP bonded bone specimens with results from the FE simulations three and four lamina bondings were sufficient. The four lamina FRAP endured $61 \%$ higher forces than those found in the FE model while for the three lamina bond it was $14 \%$. The two lamina bond would have failed during the conditions simulated in FE. With the knowledge gained from these results it would be desirable to use a four lamina fibre FRAP technology. Although this study shows promising results of bonding cervical fractures, there are limitations.

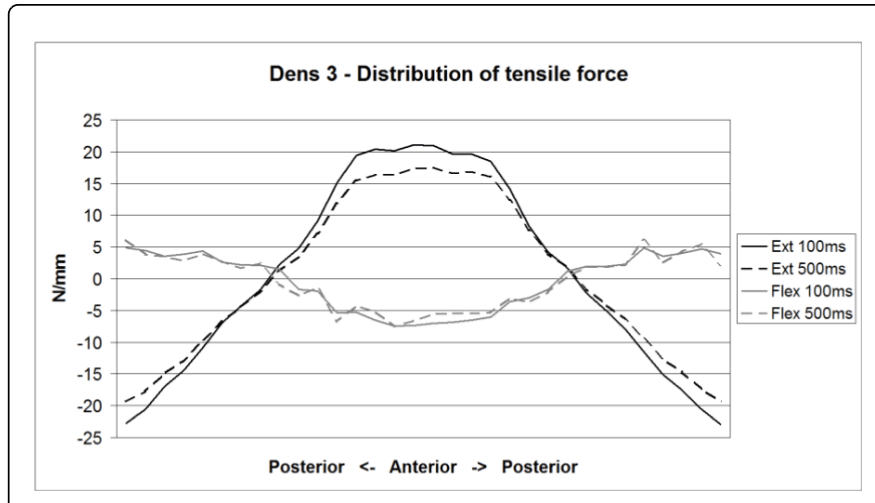

Figure 5: Distribution of tensile force circumferentially around the dens 3 fracture

\begin{tabular}{|l|l|l|l|l|l|l|}
\hline Fracture type & Extension $100 \mathrm{~ms}$ & Extension $500 \mathrm{~ms}$ & Flexion $100 \mathrm{~ms}$ & Flexion $500 \mathrm{~ms}$ & Lateral $100 \mathrm{~ms}$ & Lateral $500 \mathrm{~ms}$ \\
\hline C2 dens type 2 & 15.7 & 13.0 & 2.8 & 2.1 & 12.5 & 9.9 \\
\hline C2 dens type 3 & 21.1 & 17.6 & 4.9 & 6.1 & 9.5 & 7.8 \\
\hline C1 Jefferson & 6.5 & 4.1 & 5.7 & 5.6 & 15.0 & 13.1 \\
\hline
\end{tabular}

Table 2: Maximum tensile force per length in bonded fractures from FE simulations $(\mathrm{N} / \mathrm{mm})$

\section{FRAP bonding}

Currently, the most practical method of using fiber reinforcement is the application of woven fiber patches, because of their ease of manipulation and positioning on the cortical bone. Well-designed woven fiber systems are also known to influence the stress distribution and, depending on its orientation and alignment, are effective in altering, stopping, and redirecting the propagation of cracks. In this study, bonding was performed circumferentially around the fracture. However, in surgery, a circumferential bond may be difficult to perform due to lack of accessibility or anatomical circumstances. It is, therefore, important to be able to leave parts of the fracture without treatment. This was not tested in this study. Leaving parts of the fracture untreated is also important to minimize possible interference with fracture healing. Since fracture healing, among other mechanisms, evokes callous formation on the outside of the bone, a minimum of interfering material along the fracture is desirable [16]. In this study fractures were obtained by simply sawing the bone specimen in two pieces. This method was chosen to increase reproducibility. However, realistic fractures found in health care are often more complex in its shape and can in many cases add a certain amount of mechanical support to the stabilized fracture.

Bonding was performed on moist specimens, which also showed the best results. It was subjectively found that too wet specimen wet diluted the adhesive and dry specimen provided suboptimal adhesion. Bovine bone was chosen to prioritize reproducibility, the higher density than human bone [17] was not believed to significantly influence the results for this study.
The curing time of $3-4 \mathrm{~cm} / \mathrm{min}$ was sufficient to fully cure even deeply located laminas. It could be seen that decreasing the time between curing and testing from 4 hours to 45 minutes, decreased tensile strength by roughly $22 \%$. Extending the after-curing time to 12 hours, however, gave no significant increase in strength. A decrease in strength of $29 \%$ was also found when omitting the sanding preparation. The circumferential bonding width of $12-13 \mathrm{~mm}$ was chosen primarily to evoke cohesive failure for 2,3 and 4 lamina bonds and adhesive failure for the six lamina bond. Optimal FRAP size and adhesive overlap can be estimated by $\mathrm{L}=\mathrm{t}{ }^{*}(\sigma \mathrm{m} / \tau \mathrm{m})$, where: $\mathrm{t}=$ patch thickness, $\sigma \mathrm{m}=$ maximum stress in the patch, $\tau \mathrm{m}=$ shear stress in the patch-bone interface, (Figure 6).

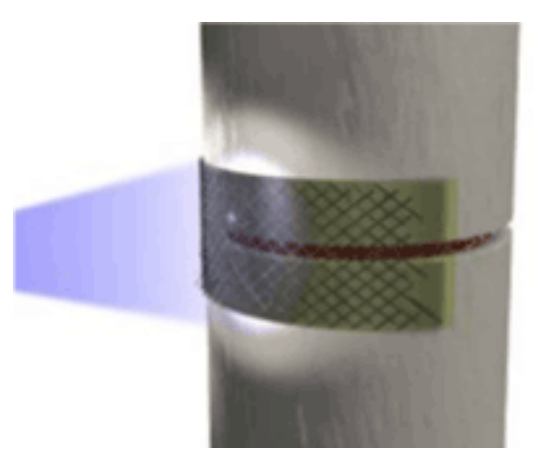

Figure 6: Circumferential application of the FRAP technology strengthened with UV light 


\section{Finite element analysis}

The KTH neck model was subjected to flexion, extension and lateral bending. Six load cases were chosen for this study, $100 \mathrm{~ms}$ and $500 \mathrm{~ms}$ dynamic loading of $5 \mathrm{Nm}$ applied to the skull. Compared to mechanical tests on cervical segments found in the literature ranging between 2-4 $\mathrm{Nm}[18-20]$ this is slightly higher. However, it was believed to be a fairly accurate and conservative approximation of mild daily loading. The $500 \mathrm{~ms}$ dynamic loading can be considered to be representative of a patient sitting with a flexed, extended or laterally bent neck. The $100 \mathrm{~ms}$ dynamic simulations are more representative of a patient subjected to mild external violence inducing a rapid neck motion. To simulate a rapid neck motion governed wilfully it would have been necessary to simulate this with active tension in the muscle elements rather than by applying an external force. The forces from the FE simulation were obtained from the isotropic shell around the induced fracture. In this study it was found that all load cases induced a major stress axis in the local $\mathrm{w}$ coordinate. Therefore, the simplification of only considering tensile forces in the local $\mathrm{w}$ coordinate was performed although this does not take into account shear and bending of the fixation bond. Since a small fracture gap was left in the FE model compressive forces were observed. However, this is in reality taken up by the surrounding bone tissue. The convergence study showed a moderate $2.51 \%$ increase of internal energies when doubling the number of elements in the vertebrae indicating a sufficient resolution of the KTH head and neck model for this study. The KTH head and neck model used in this study has been validated for local and global neck kinematics but the model has not been validated for stress prediction in the vertebrae. However, as the model has detailed geometry and has all tissues modelled anatomically it is believed that the model provides a realistic indication.

\section{Biocompatibility}

From a biocompatible point of view, Scotch bond XT is an approved dental adhesive, and thereby sufficiently biocompatible for dental use. However, for clinical use, it is unknown whether Scotch bond is non-toxic enough to ensure to not inhibit bone healing or cause any other adverse reactions. Szep et. al. [21] investigated a number of modern adhesives and found some of them to cause fibroblast apoptosis. Therefore, further studies with focus on cytotoxicity, long-term stability and amount of residual monomers, are suggested before in vivo studies are performed. Investigations regarding presence of non-polymerized, residual monomers after photo-polymerization were made by Tuusa et al. [22]. They used a bisGMA. TEGDMA-PMMA resin and found that the presence of oxygen increases residual monomers but did not find the presence of bone to increase it significantly. The biocompatibility of E-glass fibres was tested in vitro by M. Väkiparta et al. [23] who found no signs of cytotoxicity. Regarding mechanical biocompatibility, the calculated Young's modulus of 13-15GPa of the FRAP is, compared with the stiffness of human bone 15-20GPa, similar and therefore less likely to cause local stress shielding, compared to relatively stiff metal plates.

Based on the present results it is tentative to suggest that the FRAP technology has the potential to improve the surgical treatment initially of low loading fractures and later of more loading fractures in the lower part of the body. Also, it is quite possible that the FRAP technology has the potential to replace the metal implants used for skull bone flaps including bone defects in the skull bone.
Since no similar implant systems have been tested for these indications in vivo, implant related complications are yet unknown. In this study focus was to investigate mechanical sufficiency i.e. to prevent mechanical failure. However, other aspects, such as biological complications, toxicity/growth disturbances/infections etc. needs to be tested for as well in future studies. It is also important to point out that mechanical stability most likely will decrease over time. Therefore additional mechanical in vitro/vivo studies needs to be made to study this.

\section{Conclusion}

The mechanical tests with the FRAP technology was shown to exhibit sufficient strength for fixating the $\mathrm{C} 1$ and $\mathrm{C} 2$ vertebral fractures analyzed with the FE modelling. The results are encouraging and motivate further mechanical tests, FE simulations and biocompatibility tests before it is used in clinical practice.

\section{References}

1. Brolin K (2003) Neck injuries among the elderly in Sweden. Inj Control Saf Promot 10: 155-164.

2. Brolin K, von Holst H (2002) Cervical injuries in Sweden, a national survey of patient data from 1987 to 1999. Inj Control Saf Promot 9: 40-52.

3. Shao H, Bachus KN, Stewart RJ (2009) A water-borne adhesive modeled after the sandcastle glue of P. californica. Macromol Biosci 9: 464-471.

4. Gosain AK; Plastic Surgery Educational Foundation DATA Committee (2002) The current status of tissue glues: I. For bone fixation. Plast Reconstr Surg 109: 2581-2583.

5. Donkerwolcke M, Burny F, Muster D (1998) Tissues and bone adhesives--historical aspects. Biomaterials 19: 1461-1466.

6. Weber SC, Chapman MW (1984) Adhesives in orthopaedic surgery. A review of the literature and in vitro bonding strengths of bone-bonding agents. Clin Orthop Relat Res : 249-261.

7. Maurer P, Bekes K, Gernhardt CR, Schaller HG, Schubert J (2004) Tensile bond strength of different adhesive systems between bone and composite compared: an in vitro study. J Craniomaxillofac Surg 32: 85-89.

8. Atash R, Van den Abbeele A (2005) Bond strengths of eight contemporary adhesives to enamel and to dentine: an in vitro study on bovine primary teeth. Int J Paediatr Dent 15: 264-273.

9. Meechan JG, McCabe JF (1995) A comparison of the bond strengths of two different dentine-bonding agents to bone. J Oral Maxillofac Surg 53: 284-287.

10. Grossterlinden L, Janssen A, Schmitz N, Priemel M, Pogoda P, et al. (2006) Deleterious tissue reaction to an alkylene bis(dilactoyl)methacrylate bone adhesive in long-term follow up after screw augmentation in an ovine model. Biomaterials 27: 3379-3386.

11. Hoffmann B, Volkmer E, Kokott A, Augat P, Ohnmacht M, et al. (2009) Characterisation of a new bioadhesive system based on polysaccharides with the potential to be used as bone glue. J Mater Sci Mater Med 20: 2001-2009.

12. Halldin PH, Brolin K, Kleiven S, von Holst H, Jakobsson L, et al. (2000) Investigation of Conditions that Affect Neck Compression- Flexion Injuries Using Numerical Techniques. Stapp Car Crash J 44: 127-138.

13. Brolin K, Halldin P (2004) Development of a finite element model of the upper cervical spine and a parameter study of ligament characteristics. Spine (Phila Pa 1976) 29: 376-385.

14. Halliquist JO (1998) LS-DYNA Theoretical Manual. Livermore: Livermore Software Technology Corporation.

15. Kleiven S, Hardy WN (2002) Correlation of an FE Model of the Human Head with Local Brain Motion--Consequences for Injury Prediction. Stapp Car Crash J 46: 123-144. 
Citation: Pedersen K, Axel Nordberg A, Peter Halldin P, Holst HV, et al. (2014) Fiber Reinforced Adhesive Patch (FRAP): A New Technology for Minimal Invasive Treatments of Bone Fractures. J Trauma Treat S2: 007. doi: 10.4172/2167-1222.S2-007

Page 6 of 6

16. Woodward SC (1968) Physiological and biochemical evaluation of implanted polymers. Ann N Y Acad Sci 146: 225-250.

17. Curry JD (2002) Bones: Structure and Mechanics. Princeton University Press, USA.

18. DiAngelo DJ, Foley KT, Vossel KA, Rampersaud YR, Jansen TH (2000) Anterior cervical plating reverses load transfer through multilevel strutgrafts. Spine (Phila Pa 1976) 25: 783-795.

19. Mihara H, Cheng BC, David SM, Ohnari K, Zdeblick TA (2001) Biomechanical comparison of posterior cervical fixation. Spine (Phila $\mathrm{Pa}$ 1976) 26: 1662-1667.

20. Zhu Q, Ouyang J, Lu W, Lu H, Li Z, et al. (1999) Traumatic Instabilities of the Cervical Spine Caused by High-Speed Axial Compression in a Human Model: An in Vitro Biomechanical Study. Spine (Phila Pa 1976) 24: 440-444.
21. Szep S, Kunkel A, Ronge K, Heidemann D (2002) Cytotoxicity of modern dentin adhesives--in vitro testing on gingival fibroblasts. J Biomed Mater Res 63: 53-60.

22. Tuusa SMR, Puska MA, Lassila LVJ, Vallittu PK (2005) "Residual monomers released from glass-fibre-reinforced composite photopolymerised in contact with bone and blood. J Mater Sci Mater Med 16: 15-20.

23. Väkiparta M, Koskinen MK, Vallittu P, Närhi T, Yli-Urpo A (2004) In vitro cytotoxicity of E-glass fiber weave preimpregnated with novel biopolymer. J Mater Sci Mater Med 15: 69-72. 